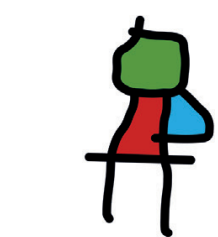

SEICAIP

\section{Allergologia et \\ immunopathologia}

Sociedad Española de Inmunología Clínica,

Alergología y Asma Pediátrica

www.all-imm.com

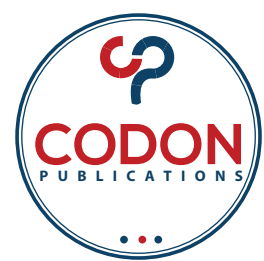

ORIGINAL RESEARCH

\title{
Downregulation of FPR1 abates lipopolysaccharide-induced inflammatory injury and apoptosis by upregulating MAPK signaling pathway in murine chondrogenic ATDC5 cells
}

\author{
Hongtao Chen ${ }^{\mathrm{a}}$, Li Zhang ${ }^{\mathrm{b} *}$
}

\author{
${ }^{a}$ Department of Orthopedics, The Sixth Affiliated Hospital of Xinjiang Medical University, Urumqi City, Xinjiang Uygur Autonomous \\ Region 830002, China \\ ${ }^{b}$ Department of Orthopedics, Wuhan Hankou Hospital, Wuhan City, Hubei Province 430012, China
}

Received 31 May 2021; Accepted 1 July 2021

Available online 1 September 2021

\section{KEYWORDS \\ apoptosis; \\ ATDC5; \\ FPR1; \\ LPS; \\ MAPK}

\begin{abstract}
Background and objective: Osteoarthritis is the most common chronic osteoarthrosis disease. There are complex factors that lead to osteoarthritis. Therefore, it is essential to investigate the molecular mechanism of osteoarthritis, especially the mechanism of articular cartilage degeneration. In this study, the mechanism of FPR1 (formyl peptide receptor 1) in LPS (lipopolysaccharide) induced chondrogenic cell ATDC5 was investigated.

Materials and methods: We employed real-time quantitative polymerase chain reaction (RT-qPCR) and western blot assay to analyze the expression level of FPR1 in ATDC5 cell lines induced by LPS at $0,2.5,5$, and $10 \mu \mathrm{g} / \mathrm{mL}$ concentrations. Then we constructed the FPR1 knockdown plasmid to transfect the LPS-ATDC5. MTT assay was used to test cell viability in control, LPS, LPS+shNC and LPS+shFPR1 groups. ELISA and RT-qPCR assay were employed to examine the TNF- $\alpha$ (tumor necrosis factor- $\alpha$ )미L- 6 and IL-1B expression level. Flow cytometry and western blot assay were employed to analyze the apoptosis of LPS-ATDC5. Finally, we utilized the western blot assay to text related protein expression level of MAPK (mitogen-activated protein kinase) signaling pathway.

Results: In this study, we found the expression level of FPR1 was increased in LPS-ATDC5, downregulation of FPR1 improves the survival rate and alleviates inflammatory response of LPS-ATDC5. Meanwhile, downregulation of FPR1 alleviates apoptosis of LPS-ATDC5. Finally, downregulation of FPR1 inhibits the MAPK signal pathway.

Conclusion: Present study revealed that FPR1 was highly expressed in LPS-induced chondrocytes ATDC5, and the downregulation of FPR1 abated the inflammatory response and apoptosis of LPS-ATDC5 cells by regulating the MAPK signaling pathway.

(c) 2021 Codon Publications. Published by Codon Publications.
\end{abstract}

${ }^{*}$ Corresponding author: Li Zhang, Department of Orthopedics, Wuhan Hankou Hospital, No. 7 Erqi Side Road, Jiang'an District, Wuhan City, Hubei Province, China. Email address: zhangli102488@163.com 


\section{Introduction}

Osteoarthritis (OA) is the most common chronic osteoarthrosis disease, and globally it is one of the major causes of pain and disability in older adults. ${ }^{1}$ As a degenerative disease, the degeneration of the articular cartilage is the main cause of osteoarthritis. ${ }^{2}$ There are many factors that contribute to cartilage degeneration, including aging, obesity, strain, trauma, and inflammatory diseases. ${ }^{3,4}$ Because of the complex factors that lead to osteoarthritis, it is essential to investigate the molecular mechanism of articular cartilage degeneration.

Articular cartilage plays an important role in arthrocentesis. Chondrocytes are unique cells in articular cartilage which are responsible for maintaining a balance between degradation and synthesis of extracellular matrix (ECM). ${ }^{5}$ Pro-inflammatory cytokines such as Interleukin-1 (IL-1) family, matrix metalloproteinases (MMP), and cyclooxygenase 2 (COX-2) contribute to the loss of major components of ECM during the degeneration of OA articular cartilage. ${ }^{6}$ In addition, apoptosis is also the direct cause of chondrocyte death. ${ }^{7}$ Therefore, it is necessary to explore the molecular mechanism of inflammatory response and apoptosis of chondrogenic cells.

Formyl peptide receptor 1 (FPR1), a member of the G-protein-coupled pattern-recognition receptor family, is mainly expressed by mammalian phagocytic leukocytes and is one of the major proteins that cause inflammation and tissue damage.8,9 FPR1 is associated with the development of many diseases. It has been reported that FPR1 gene silencing can inhibit the apoptosis of myocardial cells and cardiovascular remodeling in a rat model of ischemia reperfusion by inhibiting the MAPK signaling pathway, thereby alleviating myocardial injury. ${ }^{10}$ Wang et al. found that exogenous carbon monoxide could interfere with the activity of FPR1 by inhibiting p38 MAPK, so as to reduce the inflammatory response of progressive multiple sclerosis (PMS)-related neutrophils induced by LPS. ${ }^{11}$ FPR 1 is highly expressed in neuroblastoma and promotes its occurrence by activating the MAPK/Erk, PI3K/Akt, and P38-MAPK pathways. ${ }^{12}$ In the case of degenerative disc herniation, FPR1 activity was inhibited by a novel FPR1 antagonist, which attenuated pro-inflammatory factor expression and alleviated neuralgia response in a mouse model..$^{13}$ In osteoarthritis, FPR1 expression was found to be upregulated compared with normal tissue, and it is one of the 10 hub genes that causes arthritis. ${ }^{14}$ In addition, by targeting FPR1 with CFLFLF-PEG-64cu peptide to detect inflammatory responses associated with the onset of osteoarthritis, FPR1 could be used as a target for the diagnosis and treatment of osteoarthritis. ${ }^{15}$ However, the role of FPR1 in OA cartilage degeneration of has not been reported, and the specific molecular mechanism of FPR1 is unclear. The objective of this study was to investigate the mechanism of FPR1 in lipopolysaccharide (LPS)-induced chondrogenesis cell ATDC5.

\section{Materials and Methods}

\section{Cell culture}

The chondrogenic cell line ATDC5 was resuspended in Dulbecco's Modified Eagle Medium (DMEM) culture
(Biological Industries, Kibbutz Beit Haemek, Israel) supplemented with $10 \%$ fetal bovine serum (FBS; Biological Industries), 1\% penicillin/streptomycin (Gibco, Grand Island, USA), and 0.025- $\mu \mathrm{g} / \mathrm{mL}$ amphotericin B (Sigma-Aldrich, St. Louis, MO, USA). The cells were treated with increasing doses of LPS (0-, 2.5-, 5-, and 10- $\mu \mathrm{g} / \mathrm{mL}$ concentrations) at $37^{\circ} \mathrm{C}$ for $12 \mathrm{~h}$. The cells were cultured in a humidified $5 \%$ $\mathrm{CO}_{2}$ incubator at $37^{\circ} \mathrm{C}$.

\section{RNA extraction and real-time quantitative polymerase chain reaction (RT-qPCR)}

Total RNA was extracted from collected cells by adding trizol reagent (Invitrogen, CA, USA) according to the product manual. The purity and concentration of the extracted total RNA was examined by Nano Drop 1000 spectrophotometer (Thermo Fisher Scientific, Grand Island, USA). The Cham QTM SYBR ${ }^{\circledR}$ qPCR Master Mix (Vazyme, Nanjing, China) was applied to amplify the FPR1, tumor necrosis factor- $a$ (TNF-a), Interleukin 6 (IL-6), and IL-1B complementary DNAs (cDNAs) using the QuantStudio 6 Flex RT-PCR system (Life Technologies, Carlsbad, CA) according to the product manual. The expression values of target gene were normalized to U6 expression. The PCR primers were designed and chemically synthesized by Tsingke Technology (Beijing, China). Relative expressions of FPR1, TNF- $\alpha$, IL-6, and IL-1B in each experimental group were analyzed using the $2 \triangle \triangle \mathrm{ct}$ method. ${ }^{16,17}$ Primer sequences are exhibited in Table 1. All reactions were executed in triplicate.

\section{Cell transfection}

The FPR1 stable knockdown cell lines were constructed by lentivirus, which contained pFU-GW-009 vector (Gene Chem, China). All lentiviral vectors expressed enhanced green fluorescent protein (GFP), which was used for measuring their infection efficiency. The 293T cells were used to package the lentivirus. We found that the infection efficiency of ATDC5 cell lines was highest if the multiplicity of infection (MOI) was 200. The medium containing

Table 1 Primers for FPR1, TNF- $a, I L-6, I L-1 B$, and reference genes.

\begin{tabular}{lll}
\hline Gene & Primer & Sequence $\left(5^{\prime} \rightarrow 3^{\prime}\right)$ \\
\hline FPR1 & Forward & CATGGGAGGACATTGGCCTT \\
& Reverse & CACGGATTCTGACTGTGGCT \\
TNF-a & Forward & ATGAGCACAGAA AGCATGATC \\
& Reverse & TACAGGCTTGTCACTCGAATT \\
IL-6 & Forward & TTCCCTACTTCACAAGTC \\
& Reverse & ACTAGGTTTGCCGAGTAG \\
IL-1B & Forward & ACAGATGAAGTGCTCCTTCCA \\
& Reverse & GTCGGAGATTCGTAGCTGGAT \\
B-actin & Forward & GTGACGTTGACATCCGTAAAGA \\
& Reverse & GCCGGACTCATCGTACTCC \\
\hline
\end{tabular}


0.3-mg/mL puromycin (Thermo Fisher Scientific) was used to select stably transfected cells. Finally, the infected cells were divided into two groups: (i) Infection of control virus (shNC); and (ii) infection of FPR1 knockdown virus (shFPR1).

\section{MTT assay}

MTT assay was employed to assess the extent of cell viability. Briefly, the cells $\left(2.5 \times 10^{3}\right.$ cells/well $)$ were plated into 96-well plates in triplicate and treated with different conditions as indicated in each experiment. Following treatment, a final concentration of $0.5-\mathrm{mg} / \mathrm{mL}$ MTT solution (Beyotime, Shanghai, China) was added into each well, and the cells were incubated for another $4 \mathrm{~h}$ at $37^{\circ} \mathrm{C}$. Subsequently, the culture medium was discarded and 100$\mu \mathrm{L}$ dimethyl sulfoxide (DMSO; Sigma-Aldrich) was added for visualization. Optical density (OD) of each sample was detected at $490 \mathrm{~nm}$ through a microplate reader (BioTek, Winooski, VT, USA).

\section{Enzyme-linked immunosorbent assay (ELISA)}

TNF- $a$, IL-6, and IL-1B protein levels in LPS-ATDC5 cell lines were examined by ELISA using kits from eBioscience (Cat. No. 88-7013-88; San Diego, CA, USA) following manufacturer's instructions.

\section{Apoptosis assay}

Annexin V-PE-Cy5 Apoptosis Staining/detection kit (ab14159; Abcam, Cambridge, MA, USA) was applied to analyze cell apoptosis. Flow cytometry results were obtained from BD Accuri ${ }^{\mathrm{M}} \mathrm{C} 6$ (CA, USA). Briefly, the cells were digested, washed, and resuspended in binding buffer and cultured in dark in Annexin V-PE-Cy5-FITC. Again, the cells were kept in dark for at least $30 \mathrm{~min}$ at $37^{\circ} \mathrm{C}$ and quantified through flow cytometry.

\section{Western blot}

Briefly, cells were washed thrice in pre-cooled phosphate buffer solution (PBS), and the total protein was extracted by radioimmunoprecipitation assay (RIPA) buffer (Beyotime). Protein concentration was measured by using bicinchoninic acid (BCA) protein assay kits (CoWin Biotechnology, Jiangsu, China). Equal amounts of total proteins were separated using sodium dodecyl sulphate-polyacrylamide gel electrophoresis (SDS-PAGE). They were then transferred to the polyvinylidene difluoride membranes (PVDF; Millipore) and blocked by $5 \%$ non-fat milk at room temperature for 1 h. The protein was identified through overnight incubation at $4^{\circ} \mathrm{C}$ with the following specific primary antibodies: FPR1 (rabbit anti-FPR1 antibody, PA1-41398, 1:2000; Invitrogen), Bax (rabbit anti-Bax antibody, ab32053, 1:5000; Abcam), Bcl-2 (rabbit anti-Bcl-2 antibody, ab182858, 1:2000; Abcam), cleaved Caspase-3 (rabbit anti-cleaved Caspase-3 antibody, ab32042, 1:500; Abcam), p-ERK (rabbit anti-p-ERK antibody, ab201015, 1:1000; Abcam), ERK (rabbit anti-ERK antibody, ab32537, 1:1000; Abcam), p-p38 (rabbit anti-p-p38 antibody, 44-684G, 1:1000; Invitrogen), p38 (rabbit anti-p38 antibody, ab170099, 1:3,000; Abcam), p-JNK (rabbit anti-pJNK antibody, ab76572, 1:5000; Abcam), JNK (rabbit antiJNK antibody, ab199380, 1:2500; Abcam), B-actin (rabbit anti-B-actin antibody, ab8227, 1:3000; Abcam). The membranes were further incubated with horseradish peroxidase (HRP)-conjugated goat anti-rabbit immunoglobulin G (IgG) secondary antibody (ab205718, 1:1500; Abcam) and the bands on the membranes were visualized by the ECL chemiluminescence reagent (Beyotime). The B-actin was used to normalize the amount of analyzed samples, and protein bands were quantified by gray scale analysis through ImageJ software (National Institutes of Health).

\section{Statistical analysis}

All data are presented as mean \pm standard deviation obtained from three independent assays. Student's $t$-test was employed to calculate comparisons between two groups. GraphPad Prism 5 (GraphPad Software Inc., San Diego, CA, USA) was employed for analysis. $\mathrm{P}<0.05$ indicates statistically significant differences.

\section{Results}

\section{Expression of FPR1 was increased in LPS-ATDC5}

The FPR1 expression level was examined in chondrogenic cell line ATDC5, which was stimulated with 0-, 2.5-, 5-, and $10-\mu \mathrm{g} / \mathrm{mL}$ LPS concentrations for $12 \mathrm{~h}$. RT-qPCR result showed that the FPR1 mRNA expression level increased in an LPS dose-dependent manner (Figure 1A). Western blot assay result revealed that LPS treatment upregulated the protein expression level of FPR1 in a dose-dependent manner, which was consistent with RT-qPCR results (Figure 1B). These two results suggested that the expression of FPR1 was upregulated in LPS-ATDC5.

\section{Downregulation of FPR1 improved the survival rate of LPS-ATDC5}

The role of FPR1 in ATDC5 cell lines induced by $5-\mu \mathrm{g} / \mathrm{mL}$ LPS concentration was explored. It was observed while making a toxicity curve of LPS (see Supplementary Material, Figure S1) that the optimal concentration of 5- $\mu \mathrm{g} /$ $\mathrm{mL}$ LPS was required, and FPR1 was highly expressed after induction by $5-\mu \mathrm{g} / \mathrm{mL}$ LPS in our results as shown above. The results of Western blot (Figure 2A) and RT-qPCR (Figure 2B) assays displayed that LPS treatment notably increased the protein and mRNA expression level of FPR1 compared to control group in ATDC5 cells, and the FPR1 protein and mRNA expression level were downregulated in LPS+shFPR1 group compare to LPS+shNC group. Then MTT assay was employed to analyze cell viability in ATDC5 cells of control, LPS, LPS+shNC, and LPS+shFPR1 groups. The result demonstrated that viability of ATDC5 cells was enhanced in LPS+shFPR1 group compared to LPS+shNC group (Figure 2C). These results revealed that downregulation of FPR1 improved the survival rate of LPS-ATDC5. 


\section{Downregulation of FPR1 reduced inflammatory response of LPS-ATDC5}

In order to explore whether FPR1 mediates LPS-ATDC5 inflammatory response, the expression levels of TNF- $a$, IL-6, and IL-1B were examined in LPS-ATDC5. We found that

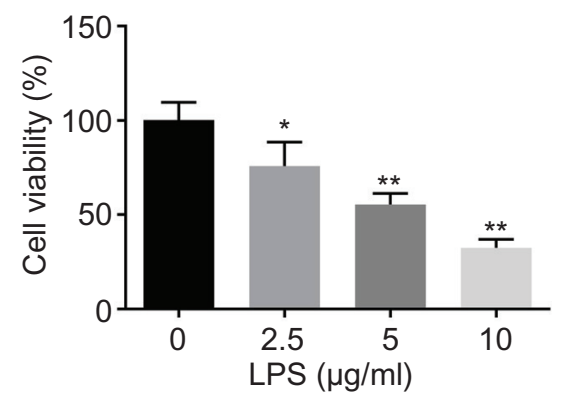

Figure S1 Toxicity of LPS on ADTC5 cells. CCK8 assay showed the cell viability of ADTC5 cells treated with LPS at 0-, 2.5-, 5-, and $10-\mu \mathrm{g} / \mathrm{mL}$ concentrations. " $\mathrm{P}<0.05,{ }^{\text {"*}} \mathrm{P}<0.01$. the concentration and mRNA expression levels of TNF-a, IL-6, and IL-1B increased notably in the LPS group compared to the control group. The ELISA and RT-qPCR results showed that both concentration of inflammatory factors and levels of genes were upregulated by the induction of LPS. The results of ELISA presented that FPR1 knockdown decreased the concentration of TNF- $a, I_{L}-6$, and IL-1B (Figure $3 A$ ). Results of RT-qPCR assay revealed that the expression levels of TNF-a, IL-6, and IL-1B were markedly decreased in LPS-ATDC5 after knocking down of FPR1 (Figure 3B). Hence, LPS-ATDC5 inflammatory response was reduced with downregulation of FPR1.

\section{Downregulation of FPR1 decreased apoptosis of LPS-ATDC5}

The apoptosis rate of LPS-ATDC5 cells was analyzed. The flow cytometry assay results revealed that the apoptosis rate increased prominently in the LPS group compared to the control group. Knockdown of FPR1 decreased the
(A)

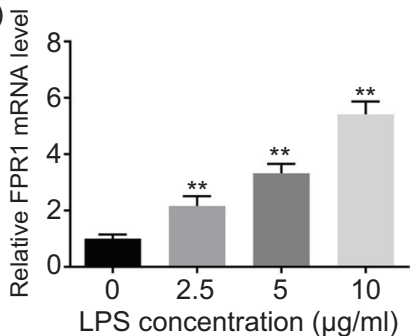

(B)

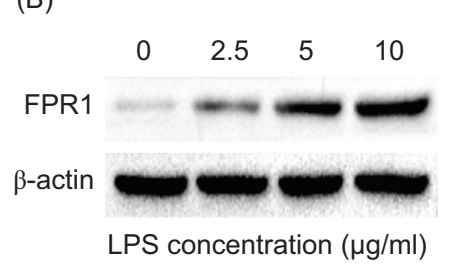

Figure 1 The expression of FPR1 was increased in LPS-ATDC5. (A) The mRNA expression level of FPR1 at 0-, 2.5-, 5-, 10- $\mu \mathrm{g} / \mathrm{mL}$ LPS concentrations; ${ }^{* *} \mathrm{P}<0.01$. (B) The protein expression level of FPR1 at 0-, 2.5-, 5-, 10- $\mu \mathrm{g} / \mathrm{mL}$ LPS concentrations; ${ }^{* *} \mathrm{P}<0.01$. $B$-actin was used as an internal control.

(A)

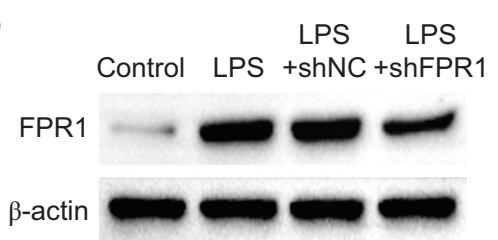

(B)

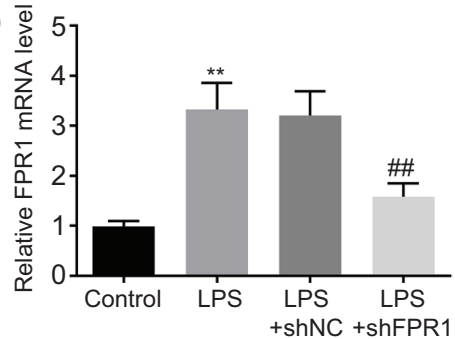

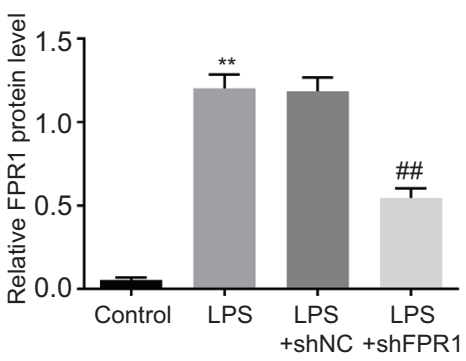

(C)

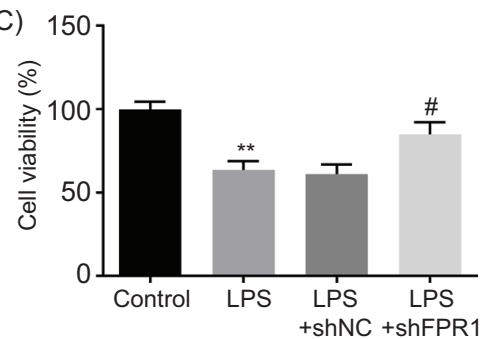

Figure 2 Downregulation of FPR1 improved the survival rate of LPS-ATDC5. (A) The protein expression level of FPR1 in ATDC5 cell of control, LPS, LPS+shNC, and LPS+shFPR1 groups; " $\mathrm{P}<0.01$. B-actin was used as an internal control. (B) The mRNA expression level of FPR1 in ATDC5 cells of control, LPS, LPS+shNC, and LPS+shFPR1 groups; ${ }^{*} P<0.01$. (C) MTT analysis of ATDC5 cell viability in control, LPS, LPS+shNC, and LPS+shFPR1 groups; ${ }^{*} \mathrm{P}<0.05,{ }^{* *} \mathrm{P}<0.01$. ${ }^{*}$ shNC versus shFPR1. 

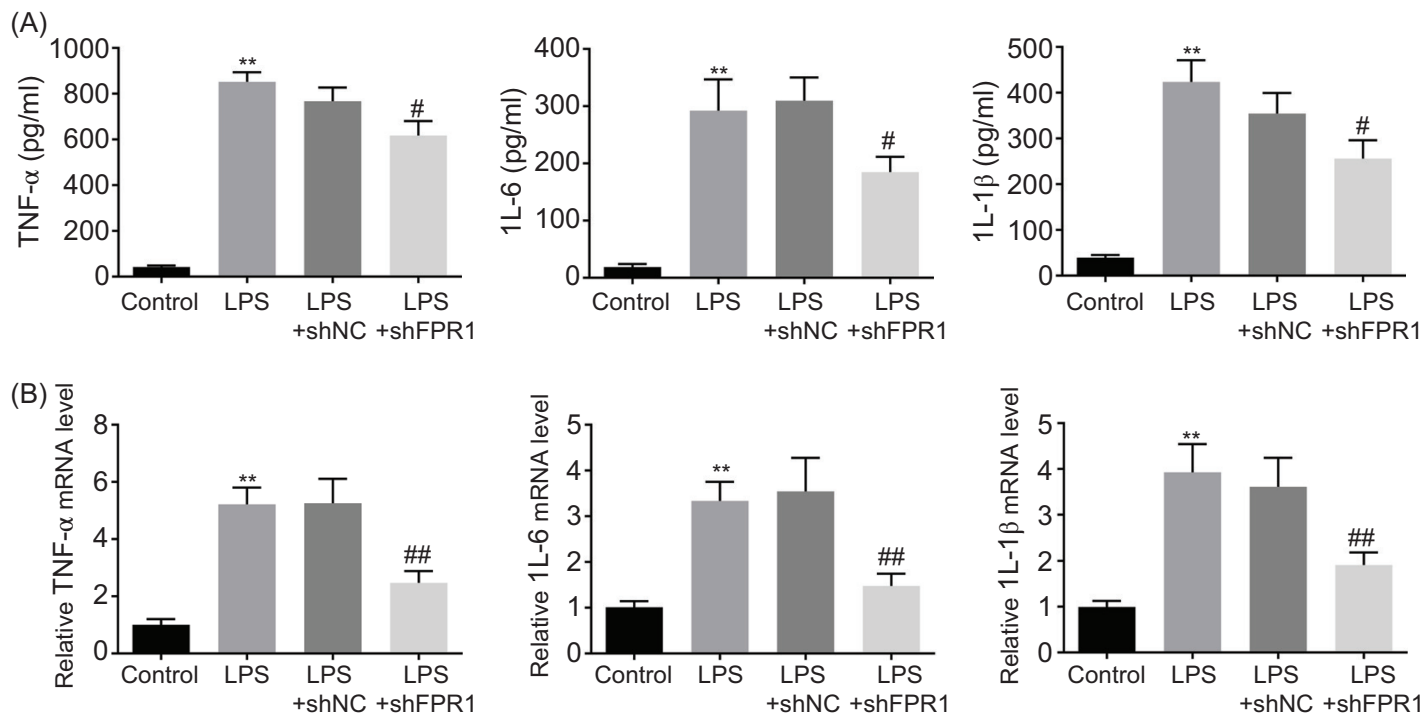

Figure 3 Downregulation of FPR1 reduced inflammatory response of LPS-ATDC5. ELISA was used to measure the concentrations of TNF-a, IL-6, and IL-1B in LPS-ATDC5 of control, LPS, LPS+shNC, and LPS+shFPR1 groups; *P $<0.05$, ${ }^{* * P}<0.01$. The (A) protein and (B) mRNA expression levels of TNF- $a$, IL-6, and IL-1B in LPS-ATDC5 of control, LPS, LPS+shNC, and LPS+shFPR1 groups. ${ }^{* *}<0.01$. \#shNC versus shFPR1.

(A)
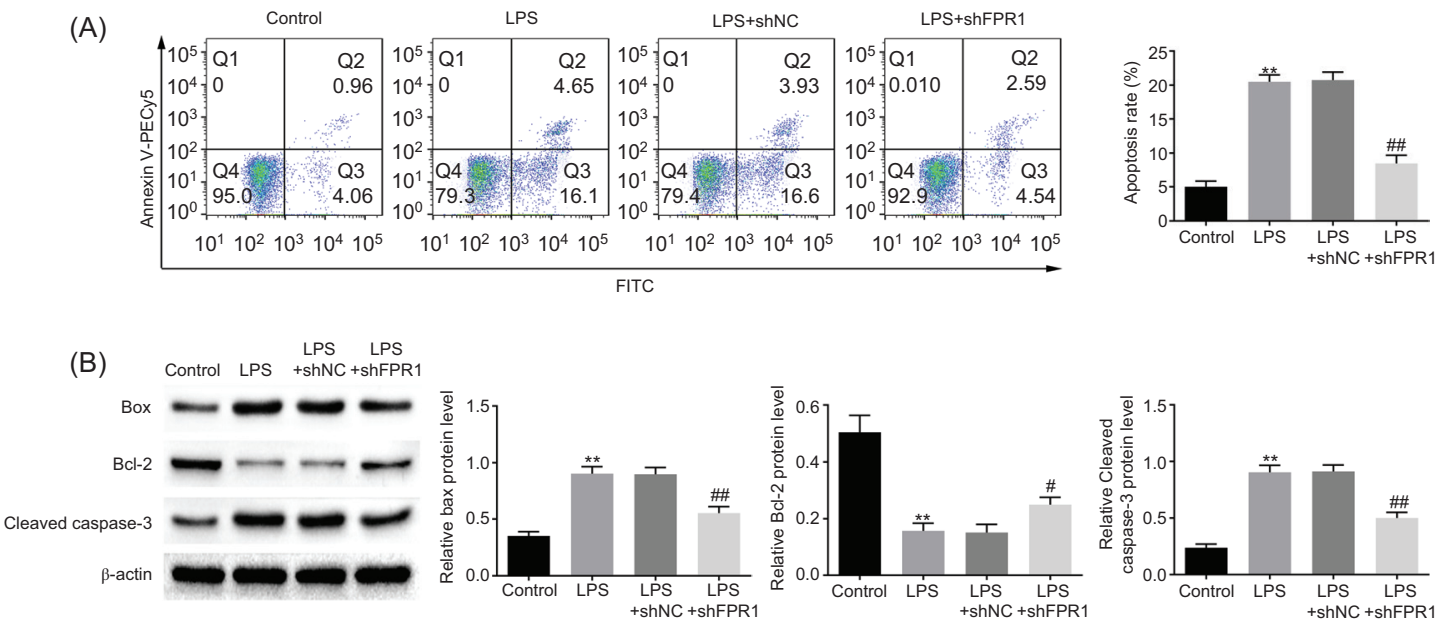

Figure 4 Downregulation of FPR1 reduced apoptosis of LPS-ATDC5. (A) The flow cytometry assay analysis of apoptosis rate. (B) Western blot analysis of Bax, cleaved Caspase-3, and Bcl-2 in Ishikawa and HEC-1-B cells after treatment of GA at 0-, 20-, 50-, $100 \mu \mathrm{M}$ concentrations. ${ }^{* *} \mathrm{P}<0.01$. GAPDH was used as an internal control. ${ }^{*}$ shNC versus shFPR1.

apoptosis rates of LPS-ATDC5 cell lines compared to the control group (Figure 4A). Further, Western blot assay was employed to examine the expression levels of apoptosis-related proteins. The results showed that after knocking down FPR1, the expression levels of Bax and cleaved Caspase- 3 were decreased whereas the expression level of Bcl-2 was upregulated in LPS-ATDC5 (Figure 4B). Collectively, these results illustrated that FPR1 knockdown decreased LPS-ATDC-5 apoptosis.

\section{Downregulation of FPR1 inhibited the MAPK signaling pathway}

Finally, the expression levels of related proteins of MAPK signaling pathway were examined by Western blot assay.
The result depicted that the activity of MAPK signaling pathway was remarkably enhanced in the LPS group compared to the control group. The expression levels of ERK, p38, and JNK all phosphorylated, were downregulated in FPR1 stable knockdown LPS-ATDC5 cells compared to the control group, while the expression levels of ERK, p38, and JNK did not change. Collectively, these results revealed that downregulation of FPR1 suppressed the MAPK signaling pathway (Figure 5).

\section{Discussion}

Osteoarthritis is the most common chronic disease of joints, affecting majority of population over the age of 65 years. It is a leading musculoskeletal cause of impaired mobility in 

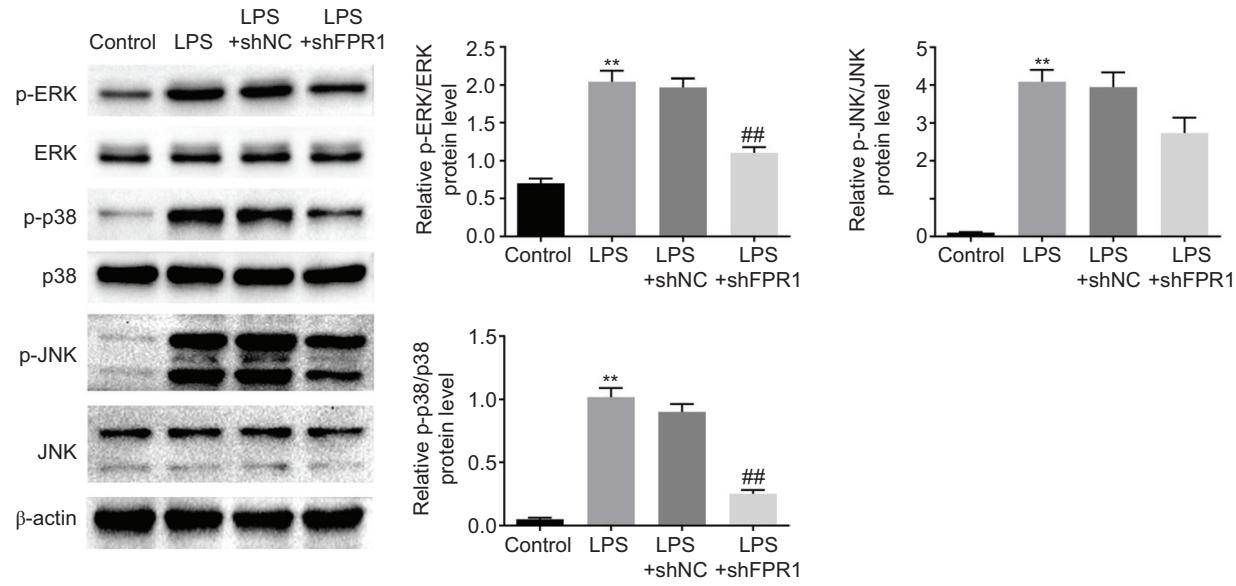

Figure 5 Downregulation of FPR1 inhibited the MAPK signaling pathway. Western blot analysis of phosphorylated ERK, ERK, phosphorylated p38, p38, phosphorylated JNK, and JNK in ATDC5 cell lines of control, LPS, LPS+shNC, and LPS+shFPR1 groups. ${ }^{*} P<0.05,{ }^{* *} P<0.01$. $\beta$-actin was used as an internal control. ${ }^{*}$ shNC versus shFPR1.

the elderly. ${ }^{18}$ Previous studies have proved that local inflammatory response leads to the pathogenesis of osteoarthritis. For example, tripterine upregulates miR-223 to relieve LPS-induced injury in osteoarthritis; ${ }^{19} \tan$ IIA protected ATDC5 cells from LPS-induced injury by reducing miR-203a expression level and inhibiting JAK/STAT and JNK pathways: ${ }^{20}$ piperine relieves LPS-induced inflammatory injury by decreasing the expression level of miR-127 in osteoarthritis; ${ }^{21}$ and pretreatment with green tea polyphenols (GTP) reduces LPS-induced inflammatory response in osteoarthritis by inhibiting the MAPK and NF-KB pathways through regulating miR-9 positively. ${ }^{22}$ Therefore, exploring effective molecules that could reduce the inflammatory response of osteoarthritis is necessary. Our study for the first time validated that FPR1 promotes the injury of osteoarthritis, suggesting that FPR1 could be involved in its pathogenesis.

FPR1 has been found to play multiple roles in many animal diseases. These roles are ambivalent, and could be beneficial or harmful. ${ }^{23}$ FPR1 has a function in the tumorigenicity of human cervical cancer. ${ }^{9}$ Suppressing FPR1 could be a potential intervention strategy to manage triple-negative breast cancer (TNBC) displaying the characteristics of breast cancer. ${ }^{24}$ Blocking FPR1 suppresses activation of neutrophil and protects against acute lung injury by targeting dipeptide $\mathrm{HCH} 6-1 .{ }^{25}$ It is found in this study that the expression level of FPR1 was increased in LPS-ATDC5, and the downregulation of FPR1 improved the survival rate, and reduced inflammatory response and apoptosis of LPS-ATDC5. Thus, FPR1 might serve as clinical therapeutic targets in many diseases.

FPR1 regulates multiple signaling pathways. FPR1 can decrease NF-KB nuclear translocation, and can regulate NLRP3 inflammasome signaling and MAPK signaling pathways. ${ }^{26}$ FPR1-knocked out mice displayed decreased acute inflammation (MAPK and NF-KB signaling pathway activation and NRLP3 inflammasome pathway induction). ${ }^{27}$ Specific members of gut microbiota stimulate FPR1 on intestinal epithelial cells to produce reactive oxygen species, causing extracellular signal-regulated kinase MAPK, and rapid phosphorylation of focal adhesion kinase (FAK). ${ }^{28}$ Consistent with the studies that established FPR1 interact with multiple signal pathways, the present study revealed that FPR1 promotes LPS-induced inflammatory injury and apoptosis through activating MAPK signaling pathway in murine chondrogenic ATDC5 cell lines. In future, it will be investigated whether FPR1 plays a role in osteoarthritis by influencing other signaling pathways.

\section{Conclusion}

It was discovered in the present study that the expression of FPR1 was increased in LPS-ATDC5. On the other hand, downregulation of FPR1 improved the survival rate of LPSATDC5, decreased the inflammatory response and apoptosis of LPS-ATDC5, and, finally, inhibited the MAPK signaling pathway. These findings suggested that downregulation of FPR1 abated LPS-induced inflammatory injury and apoptosis by upregulating the activity of MAPK signaling pathway in murine chondrogenic ATDC5 cells. However, as this study was restricted to explore the functioning of FPR1 in MAPK signaling pathway, the future research would investigate whether it works on other signal pathway as well.

\section{Funding}

This work was supported by the Natural Science Foundation of Xinjiang Uygur Autonomous Region (Grant No. 2020D01C198).

\section{Competing Interests}

The authors stated that there were no conflicts of interest to disclose.

\section{Authors' Contributions}

Hongtao Chen and Li Zhang designed the study and supervised data collection. Hongtao Chen analyzed and interpreted the data. Li Zhang prepared and reviewed the draft of the manuscript for publication. Both authors read and approved the final manuscript. 


\section{References}

1. Vega A, Martín-Ferrero MA, Del Canto F, Alberca M, García V, Munar A, et al. Treatment of knee osteoarthritis with allogeneic bone marrow mesenchymal stem cells: A randomized controlled trial. Transplantation. 2015;99(8):1681-90. https:// doi.org/10.1097/TP.0000000000000678

2. Pan L, Liu $D$, Zhao L, Wang L, Xin M, Li X. Long noncoding RNA MALAT1 alleviates lipopolysaccharide-induced inflammatory injury by upregulating micro-RNA-19b in murine chondrogenic ATDC5 cells. J Cell Biochem. 2018;119(12):10165-75. https:// doi.org/10.1002/jcb.27357

3. Fan L, He Y, Han J, Ybuan P, Guo X, Wang W. The osteoarthritis-associated gene PAPSS2 promotes differentiation and matrix formation in ATDC5 chondrogenic cells. Exp Therap Med. 2018;16(6):5190-200. https://doi.org/10.3892/ etm.2018.6843

4. Gazmuri RJ, Ayoub IM, Radhakrishnan J. NHE-1 inhibitors and erythropoietin for maintaining myocardial function during cardiopulmonary resuscitation. Signa Vitae. 2010, Oct;5(2):613. https://doi.org/10.22514/SV52.112010.1

5. Wang M, Shen J, Jin H, Im HJ, Sandy J, Chen D. Recent progress in understanding molecular mechanisms of cartilage degeneration during osteoarthritis. Ann NY Acad Sci. 2011, Dec;1240:619. https://doi.org/10.1111/j.1749-6632.2011.06258.x.

6. Schuerwegh AJ, Dombrecht EJ, Stevens WJ, Van Offel JF, Bridts CH, De Clerck LS. Influence of pro-inflammatory (IL-1 alpha, IL-6, TNF-alpha, IFN-gamma) and anti-inflammatory (IL-4) cytokines on chondrocyte function. Osteoarthritis Cartilage. 2003;11(9):681-7. https://doi.org/10.1016/ s1063-4584(03)00156-0

7. López-Armada MJ, Caramés B, Lires-Deán M, Cillero-Pastor B, Ruiz-Romero C, Galdo F, et al. Cytokines, tumor necrosis factor-alpha and interleukin-1beta, differentially regulate apoptosis in osteoarthritis cultured human chondrocytes. Osteoarthritis Cartilage. 2006;14(7):660-9. https://doi.org/ 10.1016/j.joca.2006.01.005

8. Osei-Owusu P, Charlton TM, Kim HK, Missiakas D, Schneewind O. FPR1 is the plague receptor on host immune cells. Nature. 2019;574(7776):57-62. https://doi.org/10.1038/s41586-019-1570-z

9. Cao G, Zhang Z. FPR1 mediates the tumorigenicity of human cervical cancer cells. Cancer Manage Res. 2018;10:5855-65. https://doi.org/10.2147/CMAR.S182795

10. Zhou QL, Teng F, Zhang YS, Sun Q, Cao YX, Meng GW. FPR1 gene silencing suppresses cardiomyocyte apoptosis and ventricular remodeling in rats with ischemia/reperfusion injury through the inhibition of MAPK signaling pathway. Exp Cell Res. 2018;370(2):506-18. https://doi.org/10.1016/j. yexcr.2018.07.016

11. Wang $X$, Qin $W$, Song $M$, Zhang $Y$, Sun B. Exogenous carbon monoxide inhibits neutrophil infiltration in LPS-induced sepsis by interfering with FPR1 via p38 MAPK but not GRK2. Oncotarget. 2016;7(23):34250-65. https://doi.org/10.18632/ oncotarget.9084

12. Snapkov I, Öqvist CO, Figenschau Y, Kogner P, Johnsen JI, Sveinbjørnsson B. The role of formyl peptide receptor 1 (FPR1) in neuroblastoma tumorigenesis. BMC Cancer. 2016;16:490. https://doi.org/10.1186/s12885-016-2545-1

13. Xiao L, Huang R, Zhang Y, Li T, Dai J, Nannapuneni N, et al. A new formyl peptide receptor-1 antagonist conjugated fullerene nanoparticle for targeted treatment of degenerative disc diseases. ACS Appl Materials Interfaces. 2019;11(42):38405-16. https://doi.org/10.1021/acsami.9b11783

14. Li Z, Zhong L, Du Z, Chen G, Shang J, Yang Q, et al. Network analyses of differentially expressed genes in osteoarthritis to identify hub genes. BioMed Res Int. 2019;2019:8340573. https://doi.org/10.1155/2019/8340573
15. Yang X, Chordia MD, Du X, Graves JL, Zhang Y, Park YS, et al. Targeting formyl peptide receptor 1 of activated macrophages to monitor inflammation of experimental osteoarthritis in rat. J Orthop Res. 2016;34(9):1529-38. https://doi.org/10.1002/ jor.23148

16. Nolan T, Hands RE, Bustin SA. Quantification of mRNA using real-time RT-PCR. Nature Protoc. 2006;1(3):1559-82. https:// doi.org/10.1038/nprot.2006.236

17. Michaelidou K, Tzovaras A, Missitzis I, Ardavanis A, Scorilas A. The expression of the CEACAM19 gene, a novel member of the CEA family, is associated with breast cancer progression. Int J Oncol. 2013;42(5):1770-7. https://doi.org/10.3892/ ijo.2013.1860

18. Xia B, Di C, Zhang J, Hu S, Jin H, Tong P. Osteoarthritis pathogenesis: A review of molecular mechanisms. Calcif Tissue Int. 2014;95(6):495-505. https://doi.org/10.1007/s00223-0149917-9

19. Liu $Q$, Ma H, Sun $X$, Liu B, Xiao $Y$, Pan $S$, et al. The regulatory ZFAS1/miR-150/ST6GAL1 crosstalk modulates sialylation of EGFR via PI3K/Akt pathway in T-cell acute lymphoblastic leukemia. J Exp Clin Cancer Res. 2019, May;38(1):Art. No.199. https://doi.org/10.1186/s13046-019-1208-x

20. Luan L, Liang Z. Tanshinone IIA protects murine chondrogenic ATDC5 cells from lipopolysaccharide-induced inflammatory injury by down-regulating microRNA-203a. Biomed Pharmacother. 2018;103:628-36. https://doi.org/10.1016/j. biopha.2018.04.051

21. Ren C, Liang Z. Piperine alleviates lipopolysaccharide-induced inflammatory injury by down-regulating microRNA-127 in murine chondrogenic ATDC5 cells. Biomed Pharmacother. 2018;103:947-54. https://doi.org/10.1016/j.biopha.2018.04.108

22. Zhang $Q$, Wang $Y$, Zhang $M$, Ying $H$. Green tea polyphenols attenuate LPS-induced inflammation through upregulating microRNA-9 in murine chondrogenic ATDC5 cells. J Cell Physiol. 2019;234(12):22604-12. https://doi.org/10.1002/jcp. 28826

23. Vacchelli E, Le Naour J, Kroemer G. The ambiguous role of FPR1 in immunity and inflammation. Oncoimmunology. 2020;9(1):1760061. https://doi.org/10.1080/2162402X.2020. 1760061

24. Vecchi L, Alves Pereira Zóia M, Goss Santos T, de Oliveira Beserra A, Colaço Ramos CM, França Matias Colombo B, et al. Inhibition of the AnxA1/FPR1 autocrine axis reduces MDA-MB-231 breast cancer cell growth and aggressiveness in vitro and in vivo. Biochim Biophy Acta Mol Cell Res. 2018;1865(9):1368-82. https://doi.org/10.1016/j. bbamcr.2018.06.010

25. Yang SC, Chang SH, Hsieh PW, Huang YT, Ho CM, Tsai YF, et al. Dipeptide HCH6-1 inhibits neutrophil activation and protects against acute lung injury by blocking FPR1. Free Radic Biol Med. 2017;106:254-69. https://doi.org/10.1016/j. freeradbiomed.2017.02.038

26. D’Amico $R$, Fusco $R$, Cordaro $M$, Siracusa $R$, Peritore $A F$, Gugliandolo E, et al. Modulation of NLRP3 Inflammasome through formyl peptide receptor 1 (Fpr-1) pathway as a new therapeutic target in bronchiolitis obliterans syndrome. Int J Mol Sci. 2020, Mar;21(6):2144. https://doi.org/10.3390/ijms21062144

27. Fusco R, Gugliandolo E, Siracusa R, Scuto M, Cordaro M, D'Amico R, et al. Formyl peptide receptor 1 signaling in acute inflammation and neural differentiation induced by traumatic brain injury. Biology. 2020;9(9):238. https://doi.org/10.3390/ biology 9090238

28. Alam A, Leoni G, Wentworth CC, Kwal JM, Wu H, Ardita CS, et al. Redox signaling regulates commensal-mediated mucosal homeostasis and restitution and requires formyl peptide receptor 1. Mucosal Immunol. 2014;7(3):645-55. https://doi. org/10.1038/mi.2013.84 